\title{
Explanatory models of mental disorders and treatment practices among traditional healers in Mpumulanga, South Africa
}

\author{
KR Sorsdahl', AJ Flisher',3, Z Wilson'2, DJ Stein'1 \\ 1Department of Psychiatry, University of Cape Town, Cape Town, South Africa \\ 2South African Depression \& Anxiety Group (SADAG), Johannesburg, South Africa \\ 3Research Center for Health Promotion, University of Bergen, Bergen, Norway
}

\begin{abstract}
Objective: In many traditional belief systems in Africa, including South Africa, mental health problems may be attributed to the influence of ancestors or to bewitchment. Traditional healers are viewed as having the expertise to address these causes. However, there is limited information on their explanatory models and consequent treatment practices. The present study examines traditional healers' explanatory models (EMs) and treatment practices for psychotic and non-psychotic mental illnesses. Method: 4 focus group discussions (8 healers in each group) and 18 in-depth interviews were conducted. Four vignettes were presented (schizophrenia, depression, panic and somatization) and traditional healers' views on the nature of the problem, cause, consequence, treatment and patient expectations were elicited. Results: Traditional healers held multiple explanatory models for psychotic and non-psychotic disorders. Psychotic illnesses appear to be the main exemplar of mental illness and were treated with traditional medicine, while nonpsychotic illnesses were not viewed as a mental illness at all. Additionally, traditional healers do not only use herbs and substances solely from "traditional" sources but rather have incorporated into their treatment practices modern ingredients that are potentially toxic. Conclusion: Interventions aimed at increasing the mental health literacy of traditional healers are essential. In addition, investigations of the effectiveness of traditional healer treatment for psychiatric disorders should be conducted.
\end{abstract}

Key Words: Explanatory models; Traditional healers; Mental illness; South Africa

Received: 15-09-2009

Accepted: 24-09-2009

\section{Introduction}

One way of examining the role of culture in psychiatric disorders it to elicit the explanatory models of traditional healers. ${ }^{1-3}$ This approach is based on the notion that reality is socially constructed. An explanatory model (EM) is defined by Kleinman $1988^{4}$ as the "notions about an episode of sickness and its treatment that are employed by all those engaged in the clinical process". These models are linked to particular categories of illness and reveal labels and cultural idioms for expressing the experience of illness.

Early studies in South Africa describing traditional beliefs

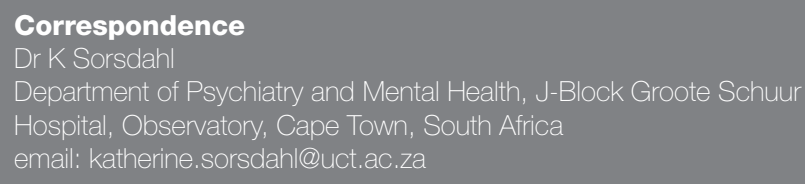

laid the groundwork for subsequent investigations into explanatory models of disease. ${ }^{5-8}$ There has been an unfortunate trend in research involving black South African psychiatric patients that has viewed the 'black experience' as being homogenous throughout the continent, irrespective of differences in class, social position, geography, language, religion and culture. ${ }^{9}$ It is not surprising that Western explanatory models have been criticized for reinforcing overt stereotypes and for romanticizing exotic accounts of African culture. ${ }^{10,11}$ It was believed that Africans would 'naturally' prefer consulting traditional healers than the more scientifically-based Western medical services. ${ }^{11}$ This claim assumes that African people had a choice of health care practitioners, when in reality this was rarely the case due to the lack of access to Western health practitioners in many parts of the country. ${ }^{12}$ However, today there are more health care options available to South Africans than in the past, making personal preference one of the 
primary drivers for consulting traditional healers. In line with this, more recent research provides evidence of a more fluid and nuanced understanding of culture. ${ }^{1,13-17}$

Although there is little data investigating explanatory models of traditional healers in South Africa, a Zimbabwean study involving traditional healers (as well as other community members) found that respondents were readily able to identify mentally ill patients based on their behaviours (e.g. wandering away from home; eating or smearing feces; laughing at inappropriate times; impaired self-care such as not washing; and eating dirty food). In order to elicit information on non-psychotic disorders, case vignettes describing typical cases of common mental disorders (CMDs) in primary care settings were presented to the care workers. Most care workers found that the vignettes did not reflect an illness, but rather suggested psychological difficulties resulting from a number of external factors (such as poverty, alcoholism, or poor marital relations). Thus, the most commonly cited causes of depression were social, spiritual or "thinking too much".

Although a few studies have been conducted in South Africa investigating traditional healers' perceptions of, and approaches to, the treatment of mental illness, none of them have specifically examined non-psychotic disorders. ${ }^{18-21}$ An understanding and appreciation of the concepts of mental illnesses held by traditional healers, as well as their treatment practices, would help plan mental health services in developing world contexts and might shed light on the debate concerning the most appropriate way to collaborate with traditional healers. It is also important for the South African public to be informed about the practices of healers in relation to mental health care so that they can make informed decisions about their choice in mental health provider.

The purpose of the present study is to identify concepts, causes, and treatments for mental disorders amongst traditional healers. More specifically we will: (a) explore traditional healers' basic concepts of mental illness including treatment; (b) contrast responses to a psychotic vignette (schizophrenia) with responses to vignettes representing non- psychotic mental disorders.

\section{Method}

\section{Setting and sample}

The South African Depression and Anxiety Group (SADAG) is a mental health advocacy group in South Africa. The group provides workshops for a variety of community members, including home-based care workers (HBCWs), educators, police, students, youth leaders and traditional healers. For this study, a convenience sample of 50 traditional healers was selected from those who attended a workshop conducted by SADAG in the province of Mpumalanga. The traditional healers were from Lydenberg, Sabi, Standerton, Bethal, Bushbuckridge and Komatipoort. According to the 2001 census, Mpumalanga has a population of 3122990 of which $92.4 \%$ are black.

\section{Procedure}

The study adopted a cross-sectional exploratory design, using qualitative methods that included 4 focus group discussions ( 8 healers in each group) and 18 in-depth interviews. The total number of participants in the study was 50. All interviews and focus groups were conducted prior to the workshops. On arrival at the community hall, traditional healers were asked to participate in the study. All of the healers approached agreed to participate.
In order to obtain information on mental disorders, four vignettes were presented (schizophrenia, depression, panic and somatization) from the Short Explanatory Model Interview, $(\mathrm{SEMI})^{22}$, a short interview used to elicit explanatory models. Traditional healers' views on the nature of the problem, cause, consequence, treatment and patient expectations were elicited. During the discussion the moderator read each vignette slowly for everyone to hear clearly and understand, and clarified anything that was uncertain before initiating the discussion. This was done in order to ensure the uniformity and clarity of the symptoms presented in the vignette, and also to allow participants who could not read the material to participate in the study. The interviews and focus groups were conducted predominately in isiZulu and Siswati using translators. All participants provided written informed consent prior to inclusion in the study. The study was approved by the Research Ethics Committee of the Health Sciences Faculty of the University of Cape Town.

\section{Data management and analysis}

The qualitative data analysis for this study was conducted using the framework approach (familiarization, identifying a thematic framework, indexing, charting, mapping and interpretation). Initially, focus group responses were read for emergent themes, which were then coded. Care was taken to ensure the codes accurately captured the respondent's meaning. A second researcher independently coded the interviews to ensure validity of the categories. We used NVivo 7.0, a qualitative software program for data analysis.

\section{Results}

Results are presented in accordance with sub-aims of this study. Results are divided into: (1) characteristics of traditional healers; (2) basic concepts of mental illness including treatment; (3) views on how schizophrenia is conceptualized and treated; (4) views on how depression is conceptualized and treated; (5) views on how panic disorder is conceptualized and treated; and (6) views on how somatization disorder is conceptualized and treated.

\section{Characteristics of traditional healers}

Table I summarizes the characteristics of the traditional healers who participated in this study. The majority of the healers interviewed were women (64\%), with an average age of 45

\begin{tabular}{|l|l|}
\hline $\begin{array}{l}\text { Table I: Socio-demographic and practice characteristics of } \\
\text { traditional healers (N=50) }\end{array}$ \\
\hline Characteristic & \\
\hline Gender (\%) & 64 \\
Female & 42 \\
Literate (\%) & 10 \\
Type of Healer (\%) & 86 \\
Herbalist & 4 \\
Diviner & $45(0.59)$ \\
Faith Healer/Diviner & $4(2.8)$ \\
Age in years (mean, sd) & $3(2.8)$ \\
Years of education (mean, sd) & $25(0.21)$ \\
Duration of training in years (mean, sd) & \\
Duration of practice in years (mean, sd) &
\end{tabular}


( $\mathrm{SD}=0.59$ ) years. A majority of the healers in this study can be classified as diviners (86\%) (who have diagnostic powers) or herbalists (10\%) (who dispense herbal medicines). $4 \%$ of the participants described themselves as being both a diviner and herbalist. Among the healers interviewed, the average education level was Grade 6 (SD = 2.8) and 58\% were illiterate. Their mean duration of practising as a traditional healer was 25 years (range: 6 months - 30 years), with an average length of training of 3 years.

\section{Basic concepts of mental illness}

All healers recognized "mental illness" as a distinct category of illness, and a majority reported having seen people with mental illness either at the time of the interview or in the previous 3 months (86\%). Although some healers (34\%) reported consulting with ancestors to aid in diagnosis, all healers reported being able to identify patients from their extreme behavioural disturbances. The more frequently reported behaviours included: violence, picking up garbage, talking randomly, walking for long periods of time and undressing in public. Descriptions of patients with mental illness include:

\begin{abstract}
"There is a lady that never even bathed herself, she didn't want to. She didn't even use the toilet and then she always wanted to go gallivanting because she hears people calling her, that are luring her towards them. She says their names and told us what they are telling her to do. She was very violent, trying to fight with people all the time."
\end{abstract}

\begin{abstract}
"Mental illness is different to stress and heart illness 'cause mental ill person will live alone, damage property and say many useless things. They do not stay at one place are very restless and talking to themselves not making any sense. They pick up dirt from dust bins, they do not wash and they beat other people up for no reason."
\end{abstract}

The healers held that mental illness could have many causes, of which witchcraft and possession by evil spirits were the most frequent (56\%). Witches were believed to have the ability to mobilize their evil powers and the forces of nature to harm other people. The term amafufunyana (12\%) was also used to describe mental illness caused by witchcraft, mostly by the Zulu-speaking respondents. Amafufunyana is a serious disorder often reported to be caused by witchcraft. Some believed that the illness was a consequence of causal factors (family problems, substance abuse, and poverty) that were left untreated and progressively became more severe (30\%). Additionally, substance abuse was mentioned as a possible cause by a few of the healers (12\%). A further belief was that when ancestors call an individual to become a traditional healer, they inflict a mental disorder on that person before the period of spirit possession begins (ukuthwasa) (10\%). An example given with regard to this cause of mental illness includes:

\footnotetext{
"The ancestors are trying to tell that person that they should follow the calling and become a traditional healer. The ancestors can cause a number of problems to a person if they do not listen to the calling, even a mental illness. A calling can be viewed as a blessing and a burden"
}

The belief in varying causes of mental illness is conveyed by the following statement:
"It is true that a person gets mentally disturbed if they don't do their family rituals, or traditions. Another person gets mad because they were abused, another because they killed someone and then you find that some people have a calling, to become traditional healer. We can help patients who have a mental illness caused by African reasons."

According to the traditional healers, the effects of a mental illness extend beyond the illness itself. A few of the healers mentioned that mentally ill people may lose their jobs, making them incapable of caring for their family (24\%). Also, may of the healers acknowledged that mentally ill patients are often ridiculed by community members (86\%) and become very lonely and isolated in their community (24\%).

However, a mentally ill person requires a great deal of caring and support, and it is usually the family who takes on this responsibility (86\%), as is portrayed by this example:
"Healing can be expensive, and families end up paying for the patient. They just want them to be better, no matter what. I had patient once whose family was desperate for cure. He would throw things at neighbours house and start fights. Other community members laugh at the sick patient too, this is very bad."

In this example, the family was held responsible for the young man's outrageous behaviour. As a result of this blame associated with mental illness, altercations develop within families and communities. This only exacerbates the financial burden placed on the family. Although the traditional healers were describing stigma, they did not use a particular word that was equivalent in meaning.

A majority of the healers reported that they possessed the skills and knowledge required to "cure a mental illness" (86\%), and often treated the patient by encouraging them to live with them in their home (90\%) or visiting them on a regular basis (98\%). The duration of this treatment ranged from two weeks to a year and a half. This ensured the patient adhered to the treatment and that someone was always there to take care of the patient. Some described situations where patients became very violent and would not take their treatment. Forceful methods such as tying them up with ropes and chains were described (34\%), in addition to getting the police involved in order to transport the patient to the clinic (12\%). The healers reported a structured treatment regime, with the patient given specific instructions to take their treatment. This is encapsulated by the following statement:

"I have a mentally ill patient, who had gone to the Western doctor and did not get cured. His family took him to me and he stayed with me for 5 months. He took 1 teaspoon of muti 3 times a day with food. Although he does not live with me anymore, he still takes his muti. He is much better now and will soon be cured". 
All of the healers reported that mentally ill patients would be given "muti" to drink and bathe with, while more than half also described treatment involving sniffing herbs through the nose (52\%). A majority of the healers could not (or would not) give precise names of the herbs that they were using to treat patients suffering from mental illness (86\%). Many of the healers claimed that they did not want to divulge such information as they considered their knowledge of plants and medicines to be inherently secretive, and were only to be shared with their initiates. However, a few were prepared to divulge this knowledge. For example, in a focus group discussion, 8 diviners asserted that the use of Dettol@ (a commercial liquid antiseptic) mixed with water and herbs was effective in treating mentally ill patients. Furthermore, one diviner provided specific details of the ingredients to cure amafufunyana. The healer claimed to be given this recipe by a talented herbalist, and has shared this knowledge with her initiates and other traditional healers.

1. 1 teaspoon Methylated spirit

2. $1 / 2$ teaspoon Benzine (colorless, highly flammable liquidused as a cleaning agent)

3. a few pieces of Indonya (a traditional herb- looks like Epsom salt)

4. 1 teaspoon of vinegar (any type)

5. $1 / 2$ teaspoon of umdlebe (you grate this particular herb)

All of these ingredients are mixed together and administered to the patient suffering from amafufunyana. The healer claimed that in only a few minutes the amafufunyana spirit begins to release. Appropriate rituals are then conducted to complete the healing process. The healer also discussed how Western practitioners would probably not approve of this treatment due to the use of Benzine. However, the healer claimed that this treatment was effective, and having the ability to cure amafufunyana allows her to become a specialist in treating this particular illness, resulting in a greater number of patients.

\section{Views on Specific Mental Illnesses \\ Schizophrenia}

Case vignette: Tshepo is 44 years old. She has not worked for years. She wears the same clothes all the time and has left her hair to grow long and untidy. She is always on her own and is often seen sitting alone and talking to herself. She is hearing voices, and believes that the government and the police are out to get her. She thinks that people are spying on her and that they know what she is thinking. Although she is polite, she does not like talking to other people. She has asked her landlord to put extra locks on her door and to remove the television set from her room. She says spies are trying to keep an eye on her because she has secret information.

Responses: A majority of the healers reported that the patient described in the "schizophrenia" vignette was not suffering from a mental illness (60\%). Some of the healers reported that the patient was being called by his ancestor to become a traditional healer (30\%). This belief is conveyed by the following statement:

"It is her ancestors that are talking to her and want her to become a traditional healer by accepting the calling. She must come see a senior traditional healer. We can help her. If she does not accept the calling things can go very bad"
The remainder reported that the patient was "thinking too much" (13\%) or was suffering from stress caused by frustration and social problems (17\%). However, on further enquiry, if told that the patient in the vignettes was also very violent and aggressive, a minority changed their mind and reported that the patient was probably suffering from a mental illness (30\%).

About 40\% of the healers in the study believed that the patient portrayed in the "schizophrenia" vignette was suffering from a mental illness. Of these healers, a majority reported that the cause of the patient's suffering was witchcraft, (25\%); some specifically used the term amafufunyana (10\%). Other healers believed the suffering of the patient in the schizophrenic vignettes was caused by their ancestors (thwasa) (10\%), or substance abuse (5\%).

\section{Agoraphobia and Panic Disorder}

Case vignette: Brian, a 34 year old taxi driver, goes to see a traditional healer. He cannot get on a bus since a friend of his was attacked at work. He has been off work for four months now. Because of this his family has money problems and they are very late paying their rent. He used to go shopping with this wife, but now does not like going into supermarkets. When he is around a lot of people he starts sweating and feels stressed and panicky. When this happens he feels that something bad is going to happen to him. He now spends much more time inside.

Responses: Almost all of the traditional healers did not believe that a man suffering with agoraphobia and panic attacks was suffering from a mental illness (98\%). However $60 \%$ did believe the individual in the vignette was suffering from an illness. The reported illnesses ranged from HIV/AIDS (16\%), illness of the heart (8\%), hypertension (12\%), ancestor calling (6\%), and stress (16\%). These illnesses were reported to require the attention of a traditional healer (42\%) or a Western doctor (16\%). All of the healers reported that they had the ability to cure these illnesses, however if they found their patients were not responding to their treatment they would refer their patient to a Western doctor.

Of the traditional healers who reported these symptoms as not being an illness (40\%), the problems reported were stress (16\%) or thinking too much (24\%). When it came to treatment, all of these traditional healers reported that there was no medical treatment available that would help someone with this disorder. However, counselling and providing practical support (money or employment) were often reported as useful alternatives.

\section{Depression}

Case vignette: Jennifer is a 29-year old single mother with two small children. They live in a small, old house that is paid for by benefits. She feels low in energy, has lost weight, does not sleep well and feels the worst in the mornings. She feels her life is not worth living and worries about what will happen to her in the future. At times, if it was not for her children she think she may end her own life. Her boyfriend pops in from time to time but is not prepared to contribute to childcare. Responses: None of the traditional healers in this study believed that the patient described in the vignette was suffering from a mental illness. A majority of the healers 
reported that these problems were caused by psychological reasons (for example, stress or thinking too much) (62\%), while others believed they may have been caused by bewitchment (12\%). Many of the healers (68\%) did believe that the patient was suffering from an illness that required the attention of a traditional healer (40\%) or a Western doctor (28\%). Among the healers that believed the patient was suffering from an illness, the reported illnesses ranged from stress (38\%), bad spirit (8\%), HIV/AIDS (8\%), illness of the heart (8\%) and thinking too much (6\%). All of these healers felt they had the capabilities of curing these patients of these problems.

Of the traditional healers who reported that the patient in the vignette was not suffering from an illness (32\%), the problems reported were stress (18\%) and thinking too much (14\%). Since the healers did not consider the patient to be ill, there was no treatment option available for them. However, some of the healers (28\%) reported that if the patient does not effectively deal with their life situation these symptoms had the potential to deteriorate and develop into a mental illness

\section{Somatization Disorder}

Case vignette: Sarah, a 45 year old machine operator married with two children has been feeling tired, a little angry and does not have much energy. She has trouble getting to sleep and she gets stomach pains and her back and legs ache most of the time. Because of this she has problems caring for her children and does not enjoy being around them like she use to. She has been to many traditional healers (and doctors). But no one can find anything wrong with her. She now wants to sit around the house watching the television.

Responses: None of the traditional healers in this study believed that the patient described in the vignette was suffering from a mental illness. A majority of the healers (74\%) believed the patient's symptoms were due to psychological problems, such as excessive worry or thinking too much, while the remainder believed the symptoms were due to a physical problem (26\%). Of the healers that believed the patient was suffering from an illness (70\%), the reported illnesses ranged from stress (30\%), physical problem (6\%), hypertension (10\%) HIV/AIDS (8\%), and thinking too much (6\%). These healers believed they had the capabilities of curing these patients.

Of the traditional healers who reported these symptoms as not being an illness (30\%), the problems reported were work related (10\%), children related (6\%) and thinking too much (12\%). These healers did not consider any treatment options for this patient, since the individual is not suffering from an illness.

\section{Discussion}

The main results of this study are as follows. Firstly, psychotic illnesses appear to be the main exemplar of mental illness, often being associated with severe behavioural disturbances. Secondly, traditional healers hold multiple explanatory models for psychotic and non-psychotic disorders. For nonpsychotic disorders, those that did not report the problem to be of a physical nature, often conceptualised these disorders as more stress-related, or a result of "thinking too much", and believed that people suffering from non-psychotic disorders did not always require treatment. Thirdly, traditional healers do not only use herbs and substances solely from "traditional" sources but rather have incorporated modern ingredients that are potentially toxic into their treatment practices.

Consistent with previous findings, healers in the present study reported severe behavioural disturbances as being associated with mentally ill patients, which can be caused by a multitude of factors. ${ }^{17,19,20,23}$ Results of the present study revealed that the symptoms presented by a mentally ill patient are predominantly behavioural and include undressing and urinating in public, violent and aggressive behaviour. Additionally, multiple causes of mental illness were described, including witchcraft, possession by evil spirits, substance abuse, life stressors and thwasa (calling to be a healer). However, according to Mzimkulu and Simbayi $(2006)^{20}$, the healers in their study reported that psychosis was differentiated from thwasa in that individuals suffering from thwasa do not present with inappropriate or bizarre behaviour, and do not have difficulty maintaining their personal hygiene. Results of the present study did not show this distinction.

Additionally, many of the healers in the present study did not believe that a patient with schizophrenia was suffering from a mental illness, but rather that their symptoms were simply a result of the patient being called by his ancestors to become a traditional healer (thwasa), "thinking too much" or suffering from stress caused by frustration and social problems. According to the healers, behaviours such as hearing voices, talking to oneself, and social withdrawal, are not always regarded as signs of a mental illness. Since a quarter of the healers altered their answer when told the patient in the vignette was violent and aggressive, it may be that traditional healers are more likely to diagnose a patient as mentally ill if they show signs of extreme antisocial behaviour or a behaviour that is not just problematic to the individual, but to the community as well.

Although, the findings of the present study support Patel's (1995) ${ }^{2}$ conclusion that psychotic illnesses appear to be the main exemplar of mental illness, according to the present study the distinction between psychotic and nonpsychotic disorders is not that straightforward. Unlike the present study, Patel's research in Zimbabwe did not elicit explanatory models of psychotic disorders using vignettes. The healers' concept of mental illness was elicited by asking the participants to simply describe mentally ill patients.

However, as traditional healers can be regarded as cultural experts, their beliefs and experiences do not necessarily coincide with the beliefs of lay people. The literature suggests that it is common for traditional healers to hear the voices of their ancestors. ${ }^{7,8}$ It may be that traditional healers were reluctant to report that the patient in the vignette was suffering from a mental illness, as they themselves may be ukuthwasa survivors and hearing the voices of ancestors informing them on how to conduct their daily lives and treat patients is an everyday occurrence. ${ }^{24}$ This could explain why many of the healers used the term thwasa to describe the patient in the schizophrenic vignette, which most reported was not suffering from a mental illness. Especially since they did not think the problems described 
in the vignettes was strange or negative. This coincides with the view of some authors that describe thwasa as a positive health experience involving the calling by the ancestors to become a traditional healer. ${ }^{25}$ However, a few of the traditional healers in this study did consider the patient with schizophrenia to be suffering from a mental illness and also used the term thwasa to describe the patient. This coincides with the suggestion that only a minority of those diagnosed with thwasa will eventually become qualified healers. If the initiate does not graduate, which also implies recovering from thwasa, they will be re-diagnosed as suffering from ukuphambana (madness). ${ }^{25}$

Although a majority of the healers did not consider the people in the non-psychotic vignettes to be suffering from a mental illness, many considered them to be suffering from other illnesses such as HIV/AIDS and hypertension. The emphasis on HIV may be an indication of HIV awareness among the population. Furthermore, since HIV is often linked to depression and other non-psychotic disorders, traditional healers may view all people experiencing these symptoms as having HIV as opposed to the non-psychotic disorders in isolation from HIV infection. Also, similar to a study conducted by Blumhagen (1980)26, the term hypertension may have been used to describe heightened psychological states of tension as opposed to a medical diagnosis of elevated blood pressure.

The traditional healers in the study hold multiple explanatory models of non-psychotic disorders. A majority of the healers regard non-psychotic disorders as a reaction to difficult life situations and as a relatively normal reaction to severe social and personal threats and losses. Therefore, a non-psychotic disorder would not be identified as a mental illness unless it acquires other characteristics such as severe behavioural disturbance. This definition of mental illness is notably different from that provided by Western medicine. These findings are relatively similar to previous studies conducted in Africa ${ }^{1,2,17,27}$ and coincide with Patel's conclusion that many Africans do not consider non-psychotic disorders to be mental disorders. ${ }^{23}$

Since many of the healers did not consider a nonpsychotic disorder to be a mental illness, many reported that there was no treatment available for these kinds of problems. However, a number of healers described more practical ways they could help these patients, such as giving them money or helping them find a job. In addition, many of the healers mentioned counseling, which could be provided by either a Western or traditional health care professional, as an option to help these patients. The healers that reported that the patients in the non-psychotic vignettes were suffering from physical disorders (such as HIV/AIDS, hypertension and other physical problems) claimed that had the skills to treat these illnesses.

In terms of investigating how traditional healers treat mentally ill patients specifically, some key preliminary findings emerged. It appears traditional healers do not solely utilize traditional herbs as ingredients for their treatment; rather they have incorporated "modern" ingredients into their practices. However, the safety of ingesting "modern" ingredients (such as methylated spirits) warrants concern. The healers may have been more willing to describe treatments that the researcher would recognize, or in other cases were afraid that the researcher would exploit their indigenous knowledge. The possibility also exists that the traditional healers themselves do not have specific knowledge of the herbs they use, such as the extent of their medicinal properties.

The results of this study have implications with regard to traditional healer practices and Western mental health services. To begin with, it would appear that traditional healers have a relatively low level of mental health literacy, and some may be misdiagnosing patients with mental illness with HIV or hypertension. Many mental illnesses, such as depression, can potentially be life threatening, and are often associated with suicide. The lifetime risk of suicide in those affected by major depression and bipolar has been estimated at $6-15 \% .{ }^{28}$ Interventions designed to increase the mental health literacy of traditional healer and to encourage referral practices for the mentally ill would be beneficial.

Secondly, collaboration between traditional healers and mental health practitioners is important with regard to effective diagnosis and treatment of mental illness. At the present time, collaborative efforts involving "Western" and traditional practitioners take on the form of a one-sided unidirectional, educative approach. There is evidence to suggest that traditional healers have shown greater influence in treating illnesses where behaviour change is required, such as HIV prevention and adherence to TB medication. ${ }^{29}$ Although formally engaging traditional healers in treating mentally ill patients may hinder to some extent appropriate diagnosis and treatment for the mentally ill, a pragmatic approach would be to work within the current structures for positive change. Traditional healers in the present study reported visiting patients with a metal illness on a daily basis or allowing them to live in their home. If these patients sought treatment from a Western medical professional, traditional healers could potentially play a pivotal role in ensuring their adherence to treatment and providing a setting where they can integrate back into the community.

Finally, the importance of investigating the effectiveness of traditional healer treatment - specifically in regard to mental illness - is gaining greater significance. All people have the right to medicines and treatment that are safe and efficacious and medicines, whether 'Western' or traditional, should fulfill the same uniform standards, tests and trials before being made available to the public. Achieving this goal is one of the main objectives of the Indigenous Knowledge Systems [Health] Lead Programme at the MRC. The program focuses on research into traditional systems of health care by evaluating the effectiveness of traditional remedies through internationally accepted scientific methods (http://www.mrc.ac.za/iks/indigenous, 2008).

\section{Limitations}

Several limitations of this study must be considered when interpreting these findings. Firstly, as this was a qualitative study that utilized a convenience sample of traditional healers from Mpumalanga, the results cannot be generalized to the South African traditional healer population. Much of the literature reported on explanatory models is from Zimbabwe or the Eastern Cape, therefore some of the differences between findings may have been due to the different settings. Secondly, the study does not provide the actual words in the African language(s) that were used to denote key concepts. Thirdly, the study was conducted in African languages, which the person 
collecting the data $(\mathrm{KS})$ does not speak. The interview schedule had to be translated from English to Zulu//Siswati/Xhosa and the responses translated back to English, creating increased opportunity for error. Also, the researcher being perceived as an outsider could have also affected the results of this study. This became apparent when trying to elicit substantial information on the treatment practices of traditional healers specifically in regard to mental illness. Finally the use of vignettes to elicit participants' beliefs on CMDs resulted in their conclusions being limited to the responses given to the cases presented and cannot be assumed to be identical to the participants' responses to real life situations

\section{Conclusion}

Despite limitations, this study has contributed to the understanding of traditional healers and mental illness in South Africa. The most important finding of this study is the low levels of mental health literacy among South African traditional healers. Furthermore, due to the potential harm of traditional healer treatment of mentally ill patients, Western health care practitioners should be advised to ask a patient if they had consulted a traditional healer before and what recommendations were made, and to discuss the implications of simultaneous traditional and Western healing interventions.

Future research should focus on replicating this study using larger samples that represent traditional healers from various regions across South Africa. Also, identification and examination of the pharmacological effects of the medicinal plants (and modern substances) used to treat mentally ill patients (as defined by the healers) should be assessed, in order to determine their use in treating mental illness and any harmful side effects that may result. Finally, training programs to increase the mental health literacy of traditional healers should be developed and the effectiveness of the program assessed.

\section{References}

1. Okello E. Cultural constructs of depression in Uganda. Unpublished doctoral thesis. 2006.

2. Patel V, Musara T, Butau T, Maramba P, Fuyane S. Concepts of mental-illness and medical pluralism in Harare. Psychological Medicine 1995; 25(3): 485-493.

3. Shankar BR, Saravanan B, Jacob KS. Explanatory models of common mental disorders among traditional healers and their patients in rural South India. International Journal of Social Psychiatry 2006; 52(3): 221-233.

4. Kleinman A. Rethinking psychiatry: from cultural category to personal experience. New York: Macmillan/The Free Press; 1988.

5. Bührmann M. Living in two worlds. Cape Town: Human \& Rousseau; 1984.

6. Cheetham RWS, Cheetham RJ. Concepts of mental-illness amongst rural Xhosa people in South-Africa. Australian and New Zealand Journal of Psychiatry 1976; 10(1): 39-45.

7. Hammond-Tooke W. Rituals and medicines: indigenous healing in South Africa. Johannesburg: AD Donker; 1989.

8. Ngubane H. Body and mind in Zulu medicine. London: Academic Press; 1977.

9. Swartz L. Culture and mental health in the rainbow nation: transcultural psychiatry in a changing South Africa. Transcultural Psychiatry 1996; 33: 119-136.

10. Swartz L, Foster D. Images of culture and mental illness: South
African psychiatric approaches. Social Dynamics 1984; 10: 17-25.

11. Swartz L. Issues for cross-cultural psychiatric research in SouthAfrica. Culture Medicine and Psychiatry 1985; 9(1): 59-74.

12. Freeman M. Mental health care in crisis in South Africa. Paper 16. Centre for Health Policy; University of the Witwatersrand; 1989.

13. Ensink K, Robertson B. Patient and family experiences of psychiatric services and indigenous healers. Transcultural Psychiatry 1999; 36: 23-43.

14. Lund C, Swartz L. Xhosa-speaking schizophrenic patients' experience of their condition: psychosis and amafufunyana. South African Journal of Psychology 1998; 28(2): 62-70.

15. Niehaus DJH, Oosthuizen P, Lochner C, Emsley RA, Jordaan E, Mbanga NI, et al. A culture-bound syndrome 'amafufunyana' and a culture-specific event 'ukuthwasa': differentiated by a family history of schizophrenia and other psychiatric disorders. Psychopathology 2004; 37(2): 59-63.

16. Alem A, Jacobsson L, Araya M, Kebede D, Kullgren G. How are mental disorders seen and where is help sought in a rural Ethiopian community? A key informant study in Butajira, Ethiopia. Acta Psychiatrica Scandinavica 1999; 100: 40-47.

17. Patel V. Explanatory models of mental-illness in Sub-Saharan Africa. Social Science \& Medicine 1995; 40(9): 1291-1298.

18. Koen L, Niehaus D, Muller J, Laurent C. Use of traditional treatment methods in a Xhosa schizophrenia population. South African Medical Journal 2003; 93(6): 443.

19. Mufamadi J. A group of traditional healers' perceptions of and approaches to the treatment of mental illness A paper presented at the indigenous knowledge conference. University of Saskatchewan: Saskatoon, Canada. University of Saskatchewan, 2001.

20. Mzimkulu J, Simbayi L. Perspectives and practices of Xhosaspeaking African traditional healers when managing psychosis. International Journal of Disability, Development and Education 2006; 53: 413-7.

21. Robertson B. Does the evidence support collaboration between psychiatry and traditional healers? Findings from three South African studies. South African Psychiatry Review 2006; 9: 87-90.

22. Lloyd KR, Jacob KS, Patel V, St Louis L, Bhugra D, Mann AH. The development of the Short Explanatory Model Interview (SEMI) and its use among primary-care attenders with common mental disorders. Psychological Medicine 1998; 28(5): 1231-1237.

23. Patel V, Simunyu E, Gwanzura F. The pathways to primary mental health care in high-density suburbs in Harare, Zimbabwe. Social Psychiatry and Psychiatric Epidemiology 1997; 32(2): 97-103.

24. Edwards SD, Grobbelaar PW, Makunga NV, Sibaya PT, Nene LM, Kunene ST, et al. Traditional Zulu theories of illness in psychiatricpatients. Journal of Social Psychology 1983;121(2): 21 3-221.

25. Schweitzer R. Categories of experience among the Xhosa: A psychological study. Unpublished master's thesis, Rhodes University, Grahamstown, South Africa 1977.

26. Blumhagen D. Hyper-tension: A folk illness with a medical name. Culture, Medicine and Psychiatry 1980;4:197-227.

27. Aidoo M, Harpham T. The explanatory models of mental health amongst low-income women and health care practitioners in Lusaka, Zambia. Health Policy and Planning 2001;16:206-13.

28. Inskip HM, Harris EC, Barraclough B. Lifetime risk of suicide for affective disorder, alcoholism and schizophrenia. British Journal of Psychiatry 1998; 172:35-7.

29. Colvin M, Gumede L, Grimwade K, Maher D, Wilkinson D. Contribution of traditional healers to a rural tuberculosis control programme in Hlabisa, South Africa. International Journal of Tuberculosis and Lung Disease 2003; 7(9): S86-S91. 